\title{
Assesment of Functional Results for the Lesions Located in Eloquent Areas with Using Intraoperative Cortical-Subcortical Stimulation and Cortical Mapping
}

\author{
Orkhan MAMMADKHANLI' ${ }^{1}$, Melih BOZKURT², Yusuf Sukru CAGLAR ${ }^{2}$ \\ ${ }^{1} Y u k s e k$ Ihtisas University, Medical Park Ankara Hospital, Department of Neurosurgery, Ankara, Turkey \\ ${ }^{2}$ Ankara University School of Medicine, Ibn-i Sina Hospital, Department of Neurosurgery, Ankara, Turkey \\ This study has been presented as an oral presentation at the $33^{\text {rd }}$ Turkish Neurosurgical Society Scientific Congress between 11 and 14 April \\ 2019 at Antalya, Turkey
}

Corresponding author: Melih BOZKURT melihbozkurt@hotmail.com

\section{ABSTRACT}

AIM: To assess the classical and functional imaging features of patients with pathology located in the eloquent areas of the brain who were admitted to our centre between October 2012 and February 2018. We also studied intraoperative somatosensory evoked potential (SEP), motor evoked potentials (MEP), phase reversal, cortical mapping, the extent of resection and the calculation of postoperative morbidity.

MATERIAL and METHODS: We compared our results with previous studies in which this technique was not used. The patient records of 163 patients were reviewed retrospectively after approval by the institutional ethics committee and comparisons were made with reports in the literature.

RESULTS: The lesion was localised in the visual cortex in eight of the 163 patients. We did not encounter any abnormality in the VEP recordings, so the surgeries were continued. The remaining 155 cases were followed by intraoperative SEP and MEP monitoring. We observed a greater than $50 \%$ decrease in the amplitude and an increase in latencies that was greater than $10 \%$ in intraoperative MEP and SEP monitoring in 24 patients of 155. Although 8 of 24 patients with abnormal SEP and MEP values were corrected with manuveurs, 6 patients developed increased neurological deficits postoperatively.

CONCLUSION: In conclusion, all of these methods should not be seen as competitive with each other; they could be considered as complementary. All of these methods are helpful for a surgical team regarding loss of neurological function. The rate of loss might be up to $100 \%$ and irreversible despite corrective maneveurs.

KEYWORDS: Eloquent area tumours, Brain mapping, Glioma surgery, Intraoperative neuromonitoring, Cortical and subcortical stimulation

\section{INTRODUCTION}

$\mathrm{S}$ urgical removal of neoplastic or non-tumoural lesions located in the central nervous system pose serious challenges to a surgical team because there is a significant risk of postoperative morbidity (disability) and mortality (death). Some areas of the brain are considered to be eloquent areas because of their tendency to result in serious disabilities after severe neurological deficits. In several studies, permanent deficit rates have been reported ranging between $13 \%$ and $20 \%(1,4,9,19,22)$.

In the literature, gross total tumour resection rates in eloquent areas have been reported to be around 40\%-50\%. The reason why this rate is too low is that surgeons prefer subtotal resection because of high postoperative morbidity 
and mortality rates. While the main principle of oncological neurosurgery is to increase survival with complete tumour excision, it should be taken into consideration that the radical removal of the mass may lead to functional loss. The aim of recent developments in the field of neurosurgery is to prevent possible morbidities after surgical resection in light of new anatomical, physiological and functional information. The aim of the newly developed sophisticated imaging technologies and intraoperative techniques is to provide extensive resection to reduce the patient's morbidity. Because the most important prognostic factor of the survival duration following surgical treatment of glial tumours, besides the histological type and grade, is the extent of resection (17).

In addition to oncological surgery, surgery on non-tumoural pathologies, such as arteriovenous malformations and cavernoma, that are located within and around the eloquent areas, are considered as high-risk surgeries. Because of surgical treatment options that provide true disease-free survival, there may become difficulties due to high morbidity and mortality rates. Therefore, other treatment options that do not provide complete palliation but less mortality and morbidity risk are evaluated first.

\section{MATERIAL and METHODS}

Our study was a retrospective study and it was conducted between October 2012 and February 2018 with the patients who applied to Ankara University Faculty of Medicine Ibn-i Sina Hospital, Department of Neurosurgery. Our study was approved by the local ethics committee (18-1119-17). A total number of 163 patients were retrospectively reviewed and the patients who had lesions in eloquent brain areas were included in our study.

Patients with diabetes mellitus, hypertension, peripheral vascular disease, peripheral neuropathy, presence of any neurological disease, previous brain surgery, neurological deficits before surgery, presence of non-eloquent area pathology and under 18 (paediatric group) were not included in our study.

The topographic relationship between the lesion and the motor area was evaluated preoperatively using computed tomography (CT) and magnetic resonance imaging (MRI) in all patients. MRI was used to define the anatomical relationship between the intracranial lesion and central sulcus using axial coronal and sagittal planes (Figure 1A, B). After intravenous gadolinium administration, the relationship between the lesions and eloquent areas, the central sulcus and other sulci that limit eloquent areas with the help of T1, T2, and contrast-weighted images were evaluated (Figure $1 \mathrm{D}-\mathrm{F}$ ). Functional MRI (fMRI; speech, motor) was used to determine the relationship between pathology and eloquent ares (Figure $1 \mathrm{C}$ ) and MR tractography (DTI) between pathology and white matter tracts.

In this study, preoperative symptoms and neurological examinations of all patients were evaluated by two neurosurgeons (O.M. M.B.). Visual examinations and computerised visual fields tests were evaluated by an ophthalmologist and auditory and audiometric examinations were evaluated by an otolaryngologist. The determination of the dominant hemisphere was performed by standardised tests. The tumour's location was precisely determined by preoperative MRI imaging. Intraoperative simultaneous functional cortico-subcortical mapping and motor mapping were performed during all surgical procedures. A prophylactic antibiotic and antiepileptic drug was administered preoperatively.

All procedures were performed under general anaesthesia. When the patients were taken to the operating table, vascular access in the left hand was obtained right after routine ASA monitorisation was obtained and fentanyl at $1 \mathrm{micr} / \mathrm{kg}$ per dose was administered for premedication. Subsequently, anaesthesia induction was achieved with propofol $(2 \mathrm{mg} / \mathrm{kg})$ and remifentanil ( $1 \mathrm{micr} / \mathrm{kg}$ ) and low dose rocuronium bromide $(0.6 \mathrm{mg} / \mathrm{kg}$ ) was used for intubation. No additional muscle relaxant drug was used following intubation. Anaesthesia was maintained with total intravenous anaesthesia. For this purpose, depth of anaesthesia was monitored by bispectral index (BIS) monitoring and titers of propofol (50-150 micr/ $\mathrm{kg} / \mathrm{min})$ and remifentanil $(0.1-1 \mathrm{micr} / \mathrm{kg} / \mathrm{min})$ were titrated to obtain BIS values between 40 and 60 . A skin flap was optimal for a planned craniotomy. Before lesion removal, a phase reversal" technique was used for central sulcus identification and cortical mapping (with strip electrode), and cortical stimulation was performed for motor cortex and sensorial cortex identification (Figure $2 \mathrm{~A}-\mathrm{C}$ ). In addition, the resection margin was optimised with cortical and subcortical direct excitations that were performed during lesion removal. As we resect deeply in the anatomical plane, the white matter stimulation was continuously maintained to not cause any damage to the connection between eloquent areas and the white matter. Surgical dissection was immediately stopped when there was a greater than $50 \%$ decrease in electrical potentials (amplitude) or a latency that was more than $50 \%$ was detected. The surgical procedure was completely stopped when no improvement was observed due to corrective manoeuvres, such as at approximately 10 minutes of waiting, $60 \mathrm{~Hz}$ interference control, and intravenous steroid administration. After the surgical procedure was completed, the same preoperative procedures were repeated and it was demonstrated that electrical activity was preserved. At the end of the surgical procedure, patients were extubated when the BIS values of the patients increased to 70 . All patients were examined as soon as they woke up for any neurological deficits, and examinations were repeated on the post-op first day, seventh day, third month, sixth month and 1 year follow-up. The severity of motor deficits was assessed with a standard motor scale;

- No deficit was considered as 0 .

- A minor deficit was considered as 1 (patient walks and uses his/her limbs almost normally but minor problems occur with fine movements in the upper extremity).

- A moderate deficit was considered as 2 (patient walks with help).

- A severe deficit was considered as 3 (patient unable to move limbs against gravity). 


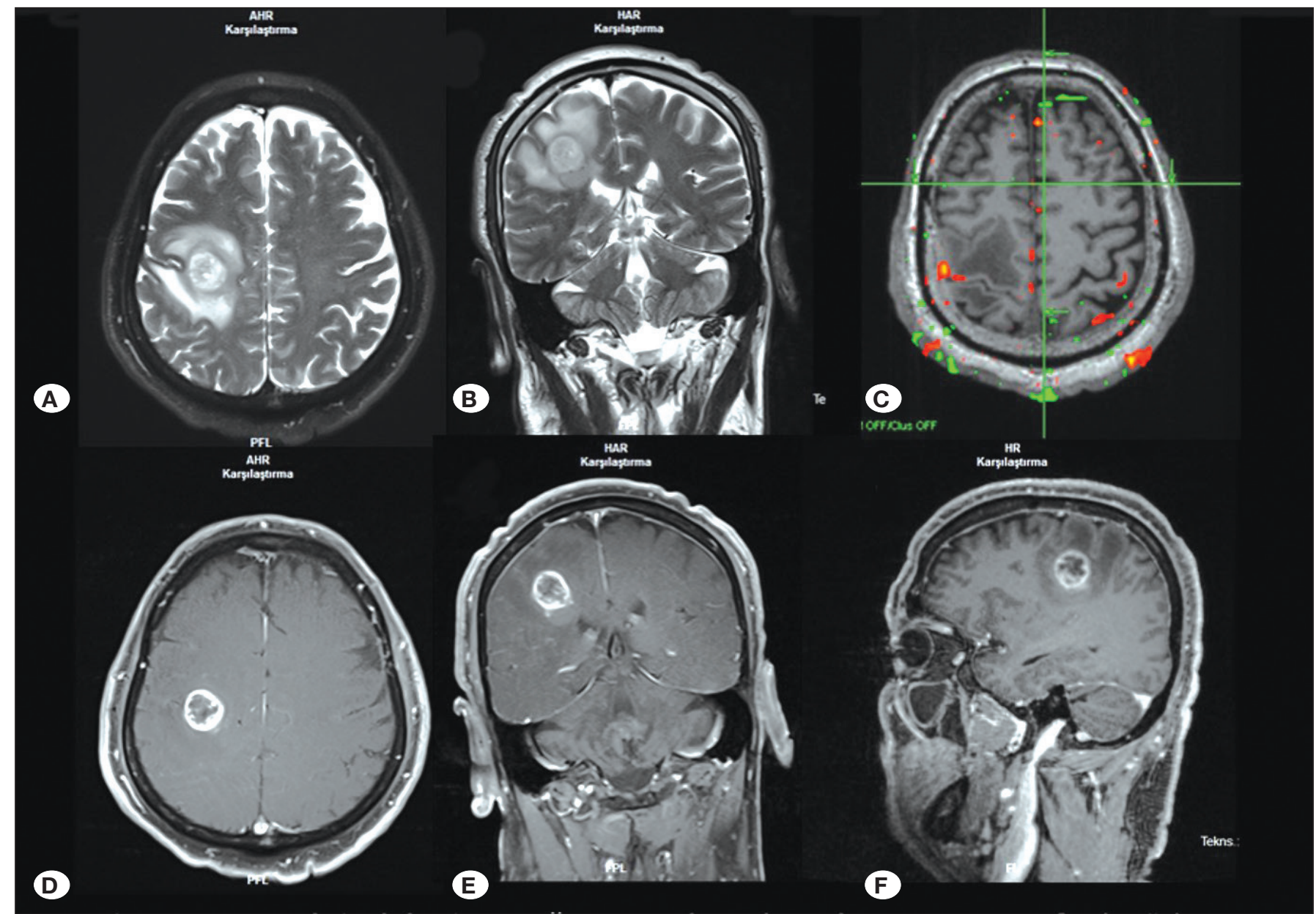

Figure 1: A) MRI with an axial view clearly shows the mass and surrounding cerebral oedema. B) Coronal view. C) Functional MRI (fMRI) shows that the mass is within the motor cortex. D) Post contrast axial MR image. E) Post contrast coronal MR image. F) Post contrast sagittal MR image. The pathology report confirmed a diagnosis of glioblastoma in this patient.
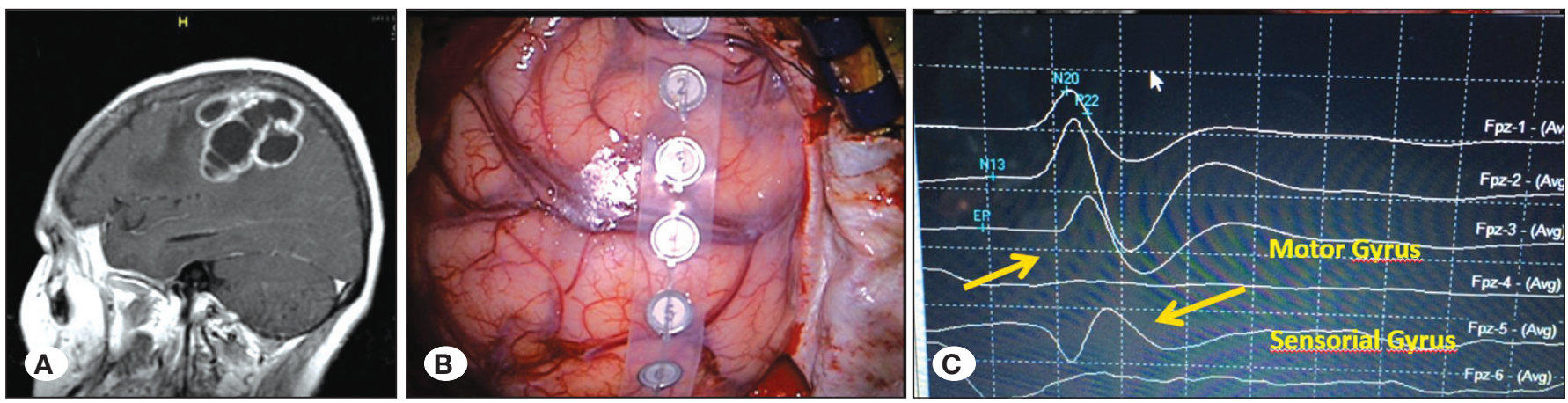

Figure 2: A) Cystic lesion that is located within the motor cortex. B) Intraoperative microelectrodes that were used to determine the central sulcus. C) Phase reversal.

All patients received a postoperative MRI on post-op Day 0 and Day 3. If the control MRI revealed no tumour then it was considered a total resection. If the residual tumour volume was less than $2 \mathrm{~cm}^{3}$ in the presence of tumours, it was considered as gross total resection. A subtotal resection was a residual tumour volume between $2-10 \mathrm{~cm}^{3}$. If the residual tumour volume was more than $10 \mathrm{~cm}^{3}$, it was considered as a partial resection or biopsy. All MRIs were evaluated by a radiologist.

\section{Statistical Analysis}

The aim of this study was to determine the variables that cause neurologic deficits in patients after brain surgery and 
to determine which variable causes morbidity after surgery. Binary logistic regression analysis was used as the analysis method in our study. The analysis was based on the data of 163 patients. As can be seen from Table VIII, there are no patients with lost data.

The table presented below gives information about how well the model performed beyond the results of the model's initial stage (Step 0). For good model performance, the Chi-squared value must be large and the corresponding probability value (p) $<0.05$. As seen in the table, the Chi-squared value with 17 degrees of freedom was 35.192 and the $p$ value was 0.0006 . According to these results, the model estimation showed successful performance.

According to Hosmer and Lemeshow test results, which showed the success of the predicted model, the model was a significant model. In the Hosmer and Lemeshow test, the probability value of the model is expected to be greater than 0.05 and the Chi-squared value is expected to be smaller. As can be seen in our results, the probability value was greater than 0.05 and Chi-squared was quite low. According to this result, the model estimation results were successful.

The Cox-Snell and Nagelkere $R^{2}$ values, which show the descriptive power of the model, indicated that the descriptive power of the model was between $20 \%$ and $35 \%$. That is, the explanatory variables included in the model explained approximately $20 \%$ or $35 \%$ of the changes in the dependent variable.

The SPSS calculations before the model estimation showed that the success of classification of the damaged and undamaged patients after the operation was $85.9 \%$. According to the information obtained from the logistic regression estimation, the logistic prediction result was more successful in separating the patients into damaged and undamaged groups. Overall, the classification success of the logistic regression estimate was $86.5 \%$.

The table below provides information on the contribution or significance of each of the independent variables in the model. Variables with a probability value less than 0.05 or 0.1 are the variables that contribute most to the description of patients with and without postoperative damage. According to the results, the gross total variable, which has a probability value of 0.073 , is the most significant and statistically significant variable for explaining the cases with and without postoperative damage. In this study, it can be said that the greater the gross total variable, the less likely there will be damage to the patient postoperatively.

\section{RESULTS}

\section{Distribution of Cases according General Characteristics}

One hundred and one of the patients were male and 62 were female, and the mean age was 48.26 (19-79). Of the patients, 139 were right-handed and 24 were left-handed. The mean follow-up period was 26.3 months (6-60 months).

\section{Distribution of Patients according Histopathological Diagnosis}

Eighty of the patients had a glial tumour. In these 80 patients, 5 patients had pilocytic astrocytoma (Grade I), 18 patients had an oligodendroglioma (Grade II), 10 patients had diffuse astrocytoma (Grade II), 9 patients had anaplastic astrocytoma (Grade III), and 38 patients had glioblastoma multiformation (Grade IV). Twenty-seven patients had meningioma and 21 patients had a metastatic brain tumour. There were 23 nontumoural patients and 10 of them had a cavernoma and 13 had arteriovenous malformations (AVMs) (Table I).

\section{Distribution of Patients according to Localisation}

Ninety-six of the patients had a lesion in the left hemisphere and 53 patients had a lesion in the right hemisphere. The two leading lesions were infiltrative and bilateral. Ten patients' lesions were in the brain stem (mesencephalon, pons, bulbus). In one patient, tumour invasion was observed to the hypothalamus and, in another patient, bilateral basal ganglion invasion was observed. In 64 of the patients, the lesion was located in the sensorimotor cortex, which is near the central sulcus. In 20 patients, the lesion was located within Broca's area and Wernicke's area. There were 33 patients with lesions in the supplementary motor area (SMA). Seven patients had a lesion in the thalamus and 15 patients had a lesion in the insular cortex (Table II).

\section{Distribution of Patients According to Resection Percentage}

In all 5 patients, the mass diagnosed as pilocytic astrocytoma was totally removed. Eighteen patients with a diagnosis of oligodendroglioma grade II received surgery, 13 patients were excised as gross total and 5 of them subtotally. Ten of the Grade II diffuse astrocytomas were excised, 4 of them as gross total and 6 of them as subtotally. Nine of the Grade III

Table I: Distribution of Patients According Histopathological Diagnosis

\begin{tabular}{lc}
\hline Histopathology & Number \\
\hline Pilocytic astrocytoma & 5 \\
\hline Oligodendroglioma (WHO II) & 18 \\
\hline Diffuse astrocytoma (WHO II) & 10 \\
\hline Anaplastic astrocytoma (WHO III) & 9 \\
\hline Glioblastoma Multiforme (WHO IV) & 38 \\
\hline Metastasis & 21 \\
\hline Arteriovenous malformation (AVM) & 13 \\
\hline Cavernoma & 10 \\
\hline Meningioma & 27 \\
\hline Others (Epandymoma, hemangioblastoma, & 12 \\
\hline melanoma, mesenchymal tumour and oth.) & 163 \\
\hline Total &
\end{tabular}


astrocytomas were excised and 4 of them were gross total and 5 of them as subtotally. Thirty-eight of the Grade IV astrocytomas were excised with 26 of them as gross total, 9 of them as subtotally, in 3 of the patients, they were partially removed. In 23 patients with vascular pathology, the lesion was totally removed. In all 21 metastatic cases, the lesion was totally removed. Twenty-one of the 27 meningiomas were removed and they were Simpson Grades I and II, 3 were removed that were Simpson Grade III and 3 were removed that were Simpson Grade IV. From 12 other classified patients, 8 of them had tumours that were removed gross totally and 4 of them were removed subtotally (Table III).

Distribution of Patients according to Changes in Motor Evoked Potentials (MEP) and Somatosensory Evoked Potential (SEP) Records

In eight of the 163 patients, the lesion was localised in the visual cortex. We did not encounter any abnormality in the

Table II: Distribution of Patients according to Localization

\begin{tabular}{lc}
\hline Localization & $\mathbf{n}$ \\
\hline Sensorimotor cortex & 64 \\
\hline Speech area (Broca's and Wernicke's areas) & 20 \\
\hline Visual & 8 \\
\hline Supplementary motor area (SMA) & 33 \\
\hline Thalamus & 7 \\
\hline Brainstem & 10 \\
\hline Basal ganglion & 5 \\
\hline Insular cortex & 15 \\
\hline Total & 163 \\
\hline
\end{tabular}

VEP recordings, so the surgeries were not stopped. The remaining 155 cases were followed by intraoperative SEP and MEP monitoring. We observed a greater than $50 \%$ decrease in amplitude and an increase in latencies of more than $10 \%$ in intraoperative MEP and SEP monitoring in 24 of 155 patients. Sixteen patients whose operations were terminated because of abnormalities in intraoperative SEP and MEP recordings showed abnormalities (amplitude and latency changes) that developed into neurological deficits postoperatively. Fourteen of these patients had lesions located in the motor cortex, one lesion was in the thalamus, three were in the insula, one in the basal ganglia, three in the brainstem and two were in the SMA. Corrective manoeuvres were performed in all of these cases, such as approximately 10 minutes standby during the operation, $60 \mathrm{~Hz}$ of interference control and intravenous steroid administration. As a result of these manoeuvres, 16 patients showed no improvement and the operation was terminated. The distribution of these 16 cases was 9 had a tumour in the motor cortex, one in the thalamus, two in the insula, one in the basal ganglia, one in the brain stem, and one in the SMA. Although 8 of 24 patients with abnormal SEP and MEP values were corrected with manuveurs, six patients developed increased neurological deficits postoperatively (Table IV, V).

\section{Distribution of Patients by Postoperative Morbidity and Mortality}

In one case in which the lesion was located in the visual cortex, a significant increase in homonym hemianopsia was observed after the operation. This increase came about six months after the operation, but complete recovery could not be achieved.

Intraoperative SEP and MEP records of all 16 patients who had the operation stopped, due to both amplitude loss and latency prolongation, showed they awakened with postoperative neurological deficits. Six of eight patients who

Table III: Distribution of Patients according to Extent of Resection

\begin{tabular}{|c|c|c|c|c|c|}
\hline Pathology & \multicolumn{4}{|c|}{ Extent of resection } & Total \\
\hline Grade II oligodendroglioma & & 13 & 5 & & 18 \\
\hline Grade II diffuse astrocytomas & & 4 & 6 & & 10 \\
\hline Grade IV astrocytomas & & 26 & 9 & 3 & 38 \\
\hline Vascular pathology (cavernoma, AVM) & 23 & & & & 23 \\
\hline Metastasis & 21 & & & & 21 \\
\hline Others & & 8 & 4 & & 12 \\
\hline Meningioma & & & & & 163 \\
\hline
\end{tabular}


had intraoperative SEP and MEP losses, which were improved after corrective manoeuvres, awakened after surgery with increased neurological deficits. Four of the 131 patients who

Table IV: Stimulation and Recording Methods of SEP and MEP

\begin{tabular}{lc}
\hline Characteristic & SEP value \\
\hline Stimulation & Cathodal stimulation \\
\hline Intensity & $21 \mathrm{~mA}$ \\
\hline Frequency & $4.71 \mathrm{~Hz}$ \\
\hline Duration & $200-300 \mu \mathrm{s}$ \\
\hline Recording & Strip/grid electrode \\
\hline Filter & $30 \mathrm{~Hz}-300 \mathrm{~Hz}$ \\
\hline Repetition & $10 \mu \mathrm{V} / \mathrm{Div}$ \\
\hline Sensivity & MEP value \\
\hline Characteristic & Anodal simulation \\
\hline Stimulation & $180 \mathrm{~V}$ \\
\hline Intensity & $500 \mathrm{~Hz}$ \\
\hline Frequency & $300 \mu \mathrm{s}$ \\
\hline Duration & $\mathrm{Needle} \mathrm{electrodes}$ \\
\hline Recording & $3 \mathrm{~Hz}-3000 \mathrm{~Hz}$ \\
\hline Filter & 5 \\
\hline Repetition & $100 \mu \mathrm{V} / \mathrm{Div}$ \\
\hline Sensivity & \\
\hline & \\
\hline
\end{tabular}

Table V: Distribution of Patients according to Changes in MEP and SEP Records

\begin{tabular}{|c|c|c|c|}
\hline & \multirow{2}{*}{$\begin{array}{l}\text { Intraoperative } \\
\text { records }\end{array}$} & \multicolumn{2}{|c|}{$\begin{array}{l}\text { Post-operative values } \\
\text { (motor power, vision) }\end{array}$} \\
\hline & & Decreased ** & $\begin{array}{c}\text { Not } \\
\text { decreased }\end{array}$ \\
\hline \multirow{2}{*}{$\begin{array}{l}\text { MEP } \\
(n=155)\end{array}$} & Changed* $^{*}(24)$ & 22 & 2 \\
\hline & Not Changed (133) & 4 & 129 \\
\hline \multirow{2}{*}{$\begin{array}{l}\text { SEP } \\
(n=155)\end{array}$} & Changed $^{*}(24)$ & 22 & 2 \\
\hline & Not changed (133) & 4 & 129 \\
\hline \multirow{2}{*}{$\begin{array}{l}\text { VEP } \\
(\mathrm{n}=8)\end{array}$} & Changed (0) & - & - \\
\hline & Not changed (8) & - & 8 \\
\hline
\end{tabular}

*We observed more than $50 \%$ decrease in amplitude and increasing in latencies more than $10 \%$ in intraoperative MEP and SEP monitoring

${ }^{* *}$ Corrective maneuvers were performed in all of these cases with loss of SEP-MEP measurements, 6 of 8 patients developed increased neurological deficits post-operatively. Despite of these corrective maneuvers, 16 patients has shown no improvement and the operation was terminated. did not have any abnormalities before the operation were awakened with postoperative neurological deficits.

In our study, in the sensorymotor cortex, brainstem, insula and thalamic region, the rate of postoperative neurological deficits was the highest (Table VI).

Surgical mortality was defined as mortality within the first 100 days postoperatively, and this was seen in two patients (Table VII).

\section{Literature Comparison}

When it comes to cases of tumours in eloquent areas, the totally and gross totally resection rates in studies have been reported as $57 \%$ for grade I and $28 \%$ for grade II tumours. In our study, it was found to be $100 \%$ and $60 \%$, respectively. Particularly, the total and postoperative morbidity rates of the tumour cases have been reported to be between $13 \%$ and $20 \%$. In our study, this rate was $14.1 \%$.

\section{DISCUSSION}

The postoperative sensory and motor deficits risk is high after surgical treatment of the central sulcus and its surrounding pathologies. There is almost no information about complete morbidity after these operations in the literature.

Safe and maximum resection for low and high grade glial tumours is necessary for long-term survival $(5,10,18,20)$. It is necessary to prevent permanent neurological deficits and to preserve sensitive areas and brain functions.

As for the operative approach, a complete preoperative plan should be performed, especially considering the MR images in axial, coronal and sagittal sections. In addition to conventional MR images, fMRI should be used in every patient. Especially in tumoural lesions, DTI images should also be included in the study to evaluate the relationship and infiltration of these lesions to the corticospinal tract, spinothalamic tract and other white matter structures. CT must be used in the presence of calcified lesions. Before surgical treatment of vascular pathologies, such as an AVM, and especially for those located within the central sulcus and nearby, DSA should be applied, and if preoperative embolisation is planned, DWI should be studied after embolisation. In this way, the radiological planning will be completed before surgical treatment of the central sulcus and its surrounding pathologies. However, all radiological methods are morphological and do not provide data on function. It should be kept in mind that morphologically defined eloquent areas may be displaced in the presence of a lesion.

The sequelae rate in non-intraoperative electrical stimulation series ranged between $13 \%$ to $27.5 \%$ and was $19 \%$ on average (8). In our study, it was $14.1 \%$.

\section{MEP in Patients with General Anaesthesia}

This method has been improved with modified electrical parameters, although many problems remain. The combined use of the motor cortex with repeated intraoperative stimulations and SSEPs may yield better results than a single 
Table VI: The Patients with Neurological Deficits after Surgery

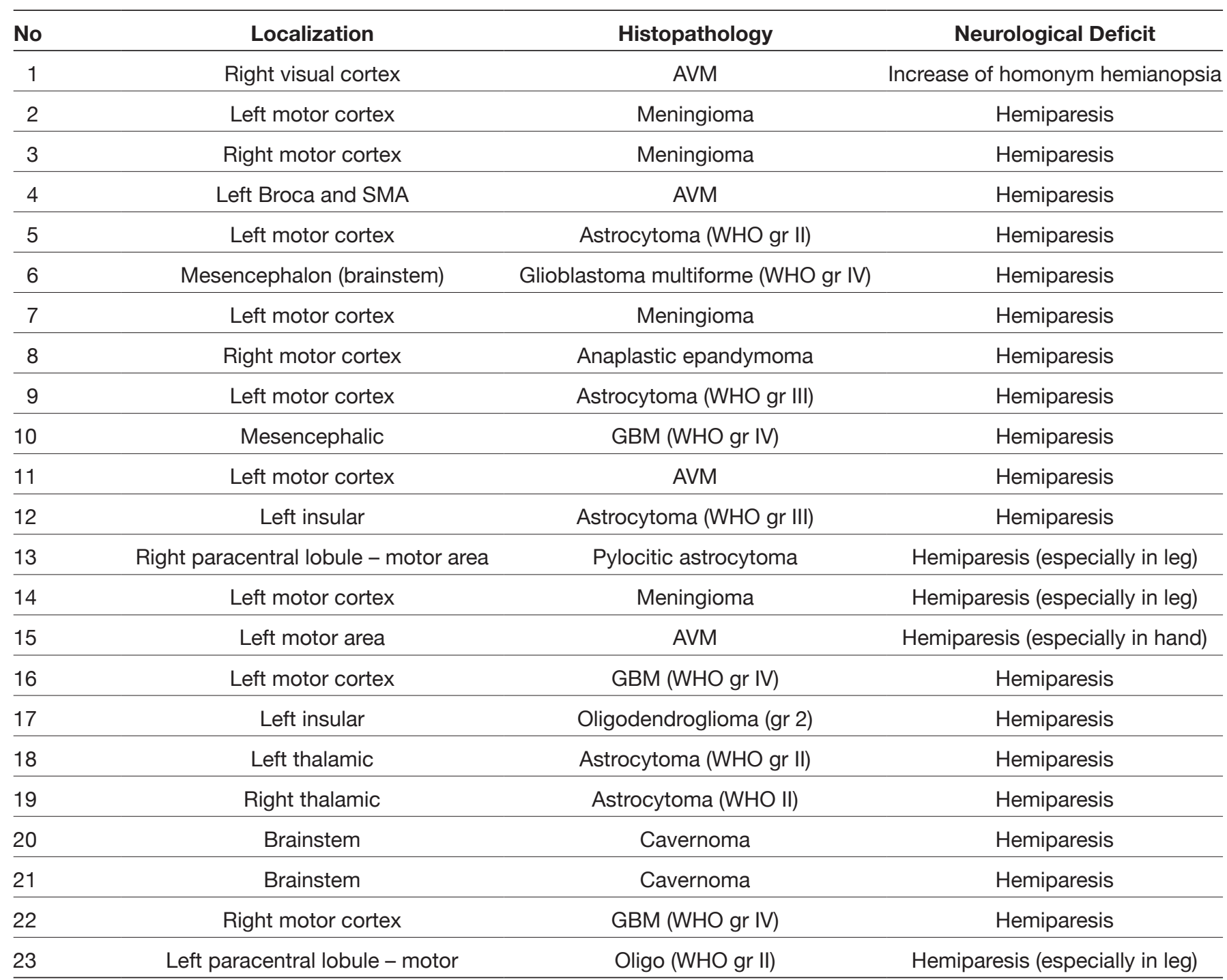

Table VII: Distribution of Patients by Postoperative Morbidity and Mortality

\begin{tabular}{lcc}
\hline \multirow{3}{*}{ Morbidity } & \multicolumn{1}{c}{$\begin{array}{c}\text { Temporary increase in } \\
\text { homonym hemianopsia }\end{array}$} & $1(0.61)$ \\
\cline { 2 - 3 } & Hemiparesis & $22(13.49)$ \\
\cline { 2 - 3 } & Total & $23(14.11)$ \\
\hline \multirow{3}{*}{ Mortality } & Hepatic insufficiency & $1(0.61)$ \\
\cline { 2 - 3 } & Fulminant meningitis & $1(0.61)$ \\
\cline { 2 - 3 } & Total & $2(1.22)$ \\
\hline
\end{tabular}

stimulation. First of all, only the monitored muscles can be controlled when recording compound muscle action potentials. Because of this, it is not possible to detect and prevent motor deficits in uncontrolled muscles. Therefore, Cedzich et al. suggested that monitoring all muscle groups at risk is necessary (4). However, it has been shown that, in the case of common low-grade gliomas involving subcortical regions where the pyramidal pathways converge, all parts of the contralateral half of the body can produce motor responses and make it difficult to predict or define all at risk muscle groups before resection. Second, as in SSEPs, even with the use of repetitive excitations, MEP provide only indirect information about the position of the pyramidal paths, and do not allow direct identification of motor paths when performing resections. Only $20 \%$ of the patients developed sequelae due to limitations of the evoked potential techniques, mainly during resection of subcortical lesions in the immediate vicinity of the pyramidal pathway. Cedzich et al. (4) concluded that an additional method is required in patients who require definitive localisation of white matter and whose motor tract tumours will be removed. 
Table VIII: Statistical Analysis of Patients

\section{Variables in the Equation}

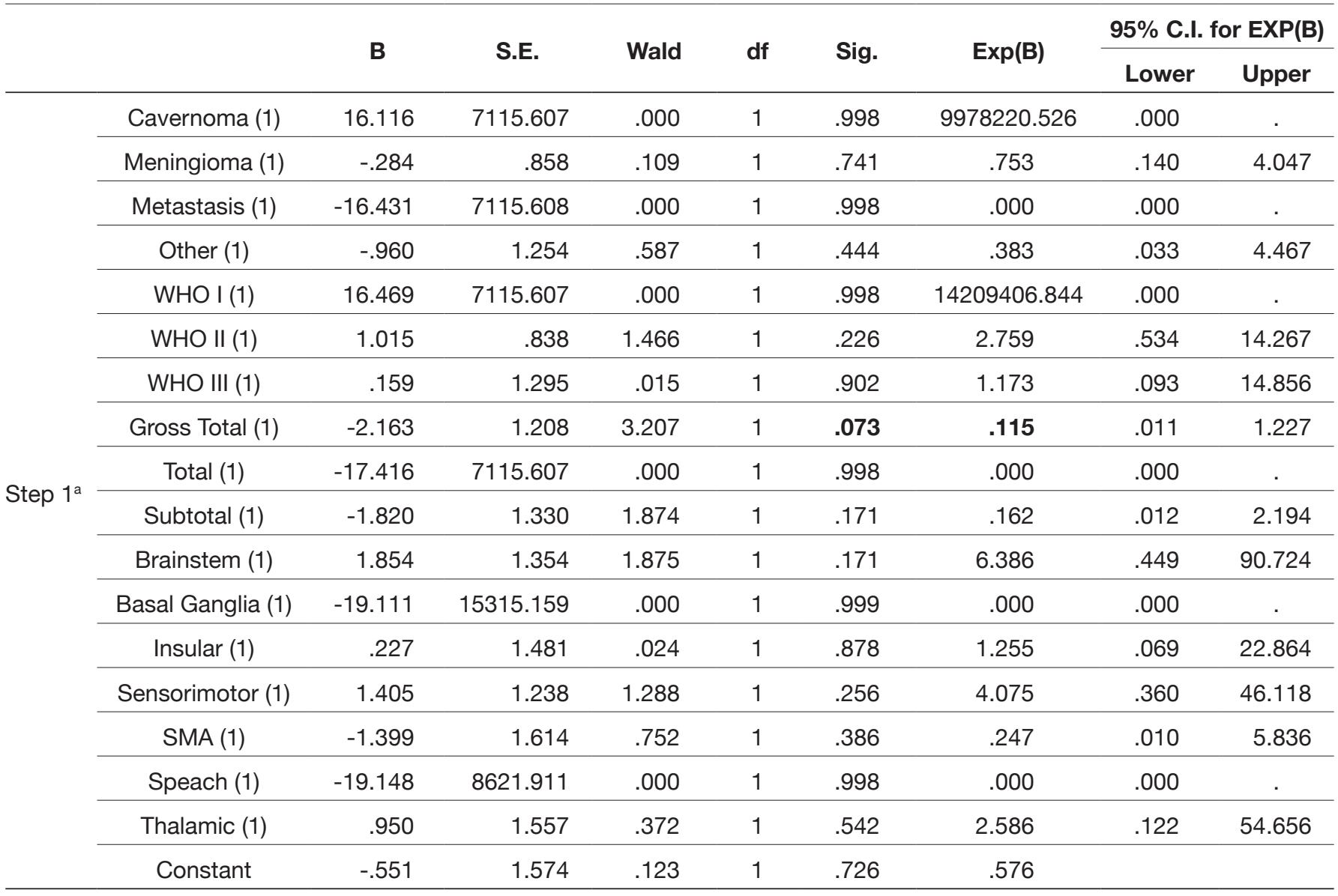

a. Variable(s) entered on step 1: Cavernoma, Meningioma, Metastasis, Other, WHO I, WHO II, WHO III, Gross Total, Total, Subtotal, Brainstem, Basal Ganglia, Insular, Sensorimotor, SMA, Speach, Thalamic.

\section{Intraoperative Repetitive Subcortical Stimulation (White Matter)}

Although the authors of numerous series reported the use of direct electrical cortical stimulation in patients after induction of general or local anaesthesia during surgery for tumours in the eloquent brain regions, there was no interest in performing intraoperative recurrent subcortical stimuli, except for Berger et al. (2,3). In conclusion, long-term and even permanent postoperative neurological deficits have been reported due to the lack of accurate and repetitive white matter mapping. In a recent study evaluating resection of a low-grade glioma close to the motor cortex, Peraud et al. reported that some patients showed severe paresis with slow recovery, possibly due to damage to the border of the white matter, when the most posterolateral portion of the tumour was removed (15). In comments regarding this article, Piepmeier confirmed that interruption of descending motor fibers is a common cause of unexpected permanent motor deficits (16). Because of this risk of damage to the pyramidal pathway at the level of deep infiltration, Peraud et al. and other investigators have concluded that it is more likely to result in permanent morbidity in resection of Grade II gliomas in the superior frontal gyrus when localisation is performed less than 0.5 $\mathrm{cm}$ from the precentral gyrus or positive stimulation points. Danks et al. studied a large group of patients with low-grade gliomas, and they described the limited resection principle at $0.5 \mathrm{~cm}$ of the primary sensorimotor cortex in a recent publication summarising all of their experiences (6). LeRoux et al. limited this line to $1 \mathrm{~cm}$ (13). Danks et al. stated that they did not use subcortical white matter stimulation mapping in their series (6), and subcortical white matter stimulation mapping was performed by Nikas et al. (14). Thus, even with a $57 \%$ complete resection rate, well-limited tumours, such as pilocytic astrocytoma, ganglioglioma, and ependymoma, have yielded gross total resection of only $28 \%$ of the true World Health Organization grade II low-grade gliomas. This rate can be optimised if there is no tumour margin around the low-grade tumour areas, but in this case, the postoperative morbidity rate increased to higher levels.

Our experience suggests that greater caution during resection in contact with functional cortico-subcortical structures and the immediate cessation of resection in the event 
of any potential change in neurophysiological recordings will reduce the rate of postoperative morbidity. This is a policy that allows improvement in the quality of low-grade glioma removal; that is, to ensure the reliability of this strategy, it is necessary to use repeated and systematic subcortical stimuli during resection. This strategy is even more important in insular region surgery. Especially in low-grade insular tumours, understanding the end of the median tumour margin poses a serious problem even for experienced surgeons. It can be challenging to the surgical team and the possibility of recovery will be very low in the presence of a possible neurologic deficit. In a series of patients with insular gliomas, Lang et al. emphasised the importance of using direct subcortical stimulation, but argued that this technique often did not provide adequate stimulation of neurological functionality and only reported loss of neurological function (12). This was also our experience. The combined use of mapping and monitoring techniques in the surgery of supratentorial lesions, especially located within the motor cortex corticospinal tract, somatosensory cortex and lemniscal pathway, allows tumour resection in previously unresectable brain regions. Further studies that analyse not only the functional outcomes but also progression of tumour recurrency and progression will be helpful to determinate the treatment plan for patients who do not undergo surgery (15).

It has been reported that the use of neuroimaging methods alone for localisation of the central sulcus may cause false localisation. Phase reversal SEP (SEP-PR) is an intraoperative mapping technique that can be used for central sulcus localisation. Cortical mapping studies performed with direct cortical stimulation and SEPs have provided great convenience to the surgical treatment in terms of the localisation of a tumour and its relation to neurological functions and increased safety of the surgery. It is not correct to use preoperative neuroimaging alone for motor cortex localisation. A study showed that the localisation with preoperative MRI was incompatible with intraoperative SEP-PR in $12 \%$ of the cases (4). As a result of craniotomy and dura opening, or during tumour excision, changes in cortical and deep structures makes it difficult to use preoperative mapping during an operation. In the 1970s, a SEP phase reversal method was developed for central sulcus localisation (21). This method is based on afferent dipole changes from the postsantral sulcus to the presantral sulcus and is actually a somatosensory potential recorded in the sensorial cortex with its mirror image recorded from the motor cortex. Therefore, SEP-PR is a safe method that can be applied noninvasively and can precisely determine the location of the central sulcus intraoperatively.

The success rate of this method is between $91 \%$ and $94 \%$ $(11,23)$. The error rate of about $9 \%$ is based on several factors; the first one is the central sulcus can be affected and mislocated by large lesions. The other reason is that the recording electrodes incorrectly positioned in the sensorimotor cortex due to the lesion can affect afferent conduction by disrupting the flow of thalamocortical pathways, changing the distribution and afferent conduction interruption in the spatial flow of the cortical electrical dipoles caused by a mass effect, and placement without considering the anatomical relationships. Generally, anaesthesia is not an important factor affecting this method because direct cortical recordings have high amplitudes and are easily observable. However, some potent anaesthetics, especially halothane, can reduce the mirror image of SEPs. Some authorities have recommended not using halothane with SEPs. The limitations of the SEP-PR technique are that they show only the central sulcus and do not allow direct display of motor functions. According to the method described by Le Roux et al., continous evaluation of motor functions during surgery is possible with direct motor cortex stimulation or white matter stimulation with bipolarity (13).

\section{CONCLUSION}

In conclusion, all of these methods should not be thought of as competetive with each other; the methods are complementary. Although all of these methods are hepful for the surgical team in terms of loss of neurological function, the rate of loss could be up to $100 \%$ and irreversible despite corrective manuveurs. Thus, despite such advanced technological equipment, high-level intraoperative imaging, navigation methods and neurophysiological recordings, a classical microsurgical technique remains important.

\section{ACKNOWLEDGEMENTS}

Preparation for publication of this article is partly supported by Turkish Neurosurgical Society.

\section{REFERENCES}

1. Apuzzo MLJ: Brain Surgery: Complication Avoidance and Management. New York: Churchill Livingstone 1993:379-390

2. Berger MS, Ojemann GA: Intraoperative brain mapping techniques in neuro-oncology. Stereotact Funct Neurosurg 58:153-161, 1992

3. Berger MS, Ojemann GA, Lettich E: Neurophysiological monitoring during astrocytoma surgery. Neurosurg Clin N Am 1:65-80, 1990

4. Cedzich C, Taniguchi M, Schäfer S, Schramm J: Somatosensory evoked potential phase reversal and direct motor cortex stimulation during surgery in and around the central region. Neurosurgery 38:962-970, 1996

5. Chaichana KL, Jusue-Torres I, Navarro-Ramirez R, Raza SM, Pascual-Gallego M, Ibrahim A, Hernandez-Hermann M, Gomez L, Ye X, Weingart JD, Olivi A, Blakeley J, Gallia GL, Lim M, Brem H, Quinones-Hinojosa A: Establishing percent resection and residual volume thresholds affecting survival and recurrence for patients with newly diagnosed intracranial glioblastoma. Neuro Oncol 16:113-122, 2014

6. Danks RA, Rogers M, Aglio LS, Gugino LD, Black PM: Patient tolerance of craniotomy performed with the patient under local anesthesia and monitored conscious sedation. Neurosurgery 42(1):28-36, 1998

7. Duffau H, Capelle L, Denvil D, Sichez N, Gatignol P, Taillandier L, Lopes M, Mitchell MC, Roche S, Muller JC, Bitar A: Usefulness of intraoperative electrical subcortical mapping during surgery for low-grade gliomas located within eloquent brain regions: Functional results in a consecutive series of 103 patients. Journal of Neurosurgery 98(4):764-778, 2003 
8. Duffau H, Lopes M, Arthuis F, Bitar A, Sichez JP, Van Effenterre R, Capelle L: Low-grade gliomas of surgery: A comparative study between two series without (1985-96) and with (19962003) functional mapping in the same institution. J Neurol Neurosurg Psychiatry 76:845-851, 2005

9. Fadul C, Wood J, Thaler H, Galicich J, Patterson RH Jr, Posner JB: Morbidity and mortality of craniotomy for excision of supratentorial gliomas. Neurology 38:1374-1379, 1988

10. Ius $T$, Isola $M$, Budai $R$, Pauletto $G$, Tomasino B, Fadiga $L$, Skrap M: Low-grade glioma surgery in eloquent areas: Volumetric analysis of extent of resection and its impact on overall survival. A single-institution experience in 190 patients. J Neurosurg 117:1039-1052, 2012

11. King RB, Schell GR: Cortical localization and monitoring during cerebral operations. J Neurosurg 67:210-219, 1987

12. Lang FF, Olansen NE, Demonte F, Gokaslan ZL, Holland EC, Kalhorn C, Sawaya R: Surgical resection of intrinsic insular tumors: Complication avoidance. Journal of Neurosurgery 95(4):638-650, 2001

13. LeRoux PD, Heyland M, Pilchner W, Ojemann GA: Use of stimulation motor mapping in the resection of intrinsic tumors from face motor cortex. Annual AANS Meeting, Nashville, April 29-May 3, 1990

14. Nikas DC, Danks RA, Black PM: Tumor surgery under local anesthesia. In: Loftus CM, Batjer HH (eds), Techniques in Neurosurgery. Philadelphia: Lippincott Williams \& Wilkins, 2001:70-84
15. Peraud A, Ansari $\mathrm{H}$, Bise $\mathrm{K}$, Reulen $\mathrm{HJ}$ : Clinical outcome of supratentorial astrocytoma WHO grade II. Acta Neurochirurgica 140:1213-1222, 1998

16. Piepmeier J, Christopher S, Spencer D, Byrne T, Kim J, Knisel JP, Lacy J, Tsukerman L, Makuch R: Variations in the natural history and survival of patients with supratentorial low-grade astrocytomas. Neurosurgery 38:872-879, 1996

17. Sanai N, Berger MS: Glioma extent of resection and its impact on patient outcome. Neurosurgery 62(4):753-764; discussion 264-266, 2008

18. Sanai N, Polley MY, McDermott MW, Parsa AT, Berger MS: An extent of resection threshold for newly diagnosed glioblastomas. J Neurosurg 115:3-8, 2011

19. Sayawa R, Hammoud M, Schoppa D, Hess KR, Wu SZ, Shi WM, Wildrick DM: Neurosurgical outcomes in a modern series of 400 craniotomies for treatment of parenchymal tumors. Neurosurgery 42:1044-1056, 1998

20. Smith JS, Chang EF, Lamborn KR, Chang SM, Prados MD, Cha S, Tihan T, VandenBerg S, McDermott MW, Berger MS: Role of extent of resection in the long-term outcome of lowgrade hemispheric gliomas. J Clin Oncol 26:1338-1345, 2008

21. Szelényi A: Intraoperative neurophysiological monitoring under general anesthesia. In: Duffau H (ed), Brain Mapping: From Neural Basis of Cognition to Surgical Applications. Springer Science \& Business Media, 2011:287

22. Tandon P, Mahapatra AK, Khosla A: Operations on gliomas involving speech centres. Acta Neurochir Suppl (Wien) 56:6771,1993

23. Wood CC, Spencer DD, Allison T, McCarthy G, Williamson PD, Goff WR: Localization of human sensorimotor cortex during surgery by cortical surface recording of somatosensory evoked potentials. J Neurosurg 68:99-111, 1988 